\title{
Effect of Temperature on Non-Destructive Wave Propagation in Uranium Monopnictides
}

\author{
R.K. Singh*, R.P. Singh, M.P. Singh and S.K. Chaurasia \\ Department of Physics, Banaras Hindu University \\ Varanasi-221005, India
}

(Received June 18, 2008; revised version October 28, 2008; in final form January 26, 2009)

\begin{abstract}
Acoustic attenuation due to phonon-phonon interaction, thermoelastic mechanism and dislocation damping were evaluated in uranium monopnictides (viz. UN, UAs and USb) in the temperature range 50-500 K, along the three crystallographic directions of propagation, viz. [100], [110] and [111] for longitudinal and shear modes of propagation. Due to antiferromagnetic property of these compounds ultrasonic attenuation due to magnon-phonon interaction was also obtained. The second- and third-order elastic moduli of B1-type uranium monopnictides were obtained using electrostatic and the Born repulsive potentials. Gruneisen numbers and acoustic coupling constants were evaluated for longitudinal and shear waves along different directions of propagation and polarization. Results were discussed and compared with available data. It was found that the temperature dependence of attenuation due to phonon-phonon interaction and thermoelastic loss mechanisms follow the third and fourth order polynomial fit laws, respectively, and acoustic attenuation is mainly governed by phonon-phonon interaction in this temperature range.
\end{abstract}

PACS numbers: 43.35.Cg, 62.20.de, 62.20.D-

\section{Introduction}

The NaCl-type uranium monopnictides (UX, $\mathrm{X}={ }^{7} \mathrm{~N}$, ${ }^{33} \mathrm{As},{ }^{51} \mathrm{Sb}$ ) exhibit a great variety of anomalous behavior as far as their elastic, electronic, lattice dynamical and magnetic properties [1-11] are concerned. Uranium monopnictides show antiferromagnetic ordering at $T_{\mathrm{N}}$ or $T_{\mathrm{C}}(53,124$, and $213 \mathrm{~K}$ for UN, UAs, and USb, respectively), which increases with increasing anion atomic number. The electronic structure of uranium monopnictides is characterized by $5 f$ and $6 d$ electrons in addition to conduction electrons close to the Fermi energy. These groups of electrons give the compounds, their metallic character and high electronic specific heat $[12,13]$. The lattice dynamical properties for these compounds have been studied using rigid ion and shell models [7], three-body force rigid ion model [8] and three-body force shell model [9]. But results on acoustical studies are not available for these compounds.

Different causes can be attributed to the attenuation of acoustic wave propagating through solids. Some important causes of acoustical dissipation are: electronphonon interaction, phonon-phonon interaction, thermoelastic loss, losses due to lattice imperfections (viz. dislocation damping due to screw and edge dislocations), and in ferromagnetic and antiferromagnetic materials,

* corresponding author; e-mail: rksingh_17@rediffmail.com magnon-phonon interaction also produces acoustical dissipation in lower temperature range $(T<50 \mathrm{~K})$. In dielectric crystals, interaction of acoustic phonons and thermal phonons is the dominant cause of acoustical dissipation at higher temperatures [14], while in ferromagnetic and antiferromagnetic compounds, in presence of external magnetic field, magnon-phonon interaction is the principal cause in the lower temperature range [15]. Such types of studies are essential for assessing the suitability of materials for several practical problems, such as the design of optimum sound transmission systems for ultrasonic delay lines [16]. From microstructure characterization point of view, it is well known that acoustic properties of materials are very important. Therefore, in the present paper we have studied the acoustic attenuation due to different causes. Second and third order elastic moduli, the Grüneisen parameters, acoustic coupling constants, and viscous drag coefficients due to screw and edge dislocations have also been evaluated for uranium monopnictides.

\section{Theoretical approach}

\subsection{Elastic moduli}

Elastic moduli of $n$-th order is defined as [17]:

$$
C_{i j k l m n \ldots}=\left(\partial^{n} u / \partial \varepsilon_{i j} \partial \varepsilon_{k l} \partial \varepsilon_{m n} \ldots\right) \text {, }
$$

where $u$ is the crystal free-energy density and $\varepsilon_{i j}$ is the strain tensor. 
Second- and third-order elastic moduli (SOEM and TOEM) at absolute zero temperature viz. $C_{i j}^{0}$ and $C_{i j k}^{0}$ are given as [18]:

$$
\begin{aligned}
& C_{11}^{0}=1.56933 \frac{z^{2} e^{2}}{r_{0}^{4}}+\frac{1}{q r_{0}}\left(\frac{1}{r_{0}}+\frac{1}{q}\right) Q\left(r_{1}\right) \\
& +\frac{2}{q r_{0}}\left(\frac{1}{\sqrt{2} r_{0}}+\frac{1}{q}\right) Q\left(r_{2}\right), \\
& C_{12}^{0}=C_{44}^{0}=0.344778 \frac{z^{2} e^{2}}{r_{0}^{4}}+\frac{1}{q r_{0}}\left(\frac{1}{\sqrt{2} r_{0}}+\frac{1}{q}\right) Q\left(r_{2}\right), \\
& C_{111}^{0}=10.26390 \frac{z^{2} e^{2}}{r_{0}^{4}}-\left(\frac{3}{q r_{0}^{2}}+\frac{3}{q^{2} r_{0}}+\frac{1}{q^{3}}\right) Q\left(r_{1}\right) \\
& -\left(\frac{3}{\sqrt{2} r_{0}^{2} q}+\frac{3}{q^{2} r_{0}}+\frac{\sqrt{2}}{q^{3}}\right) Q\left(r_{2}\right), \\
& C_{112}^{0}=C_{166}^{0}=-1.209625 \frac{z^{2} e^{2}}{r_{0}^{4}} \\
& -\left(\frac{3}{2 \sqrt{2} r_{0}^{2} q}+\frac{3}{q^{2} r_{0}}+\frac{\sqrt{2}}{q^{3}}\right) Q\left(r_{2}\right), \\
& C_{123}^{0}=C_{144}^{0}=C_{456}^{0}=0.678375 \frac{z^{2} e^{2}}{r_{0}^{4}},
\end{aligned}
$$

where $Z$ is atomic number, $r$ is nearest neighbor distance, $q$ is hardness parameter

$$
Q\left(r_{1}\right)=A \exp \left(-\frac{r_{0}}{q}\right)
$$

and

$$
Q\left(r_{2}\right)=A \exp \left(-\frac{r_{0} \sqrt{2}}{q}\right) \text {. }
$$

Second- and third-order elastic moduli at temperature, $T$ are the sum of elastic moduli at absolute zero temperature and vibrational contribution to second- and third-order elastic moduli at corresponding temperature [19-22] viz. $C_{i j}^{0}$ and $C_{i j k}^{0}$, i.e.

$$
\begin{aligned}
& C_{i j}(T)=C_{i j}^{0}+C_{i j}^{\mathrm{vib},}, \\
& C_{i j k}(T)=C_{i j k}^{0}+C_{i j k}^{\mathrm{vib} .},
\end{aligned}
$$

where $C_{i j}^{\text {vib. }}$ and $C_{i j k}^{\text {vib. }}$ are vibrational contribution to elastic moduli given by

$$
\begin{aligned}
& C_{11}^{\text {vib. }}=f^{1,1} G_{1}^{2}+f^{2} G_{2}: C_{12}^{\text {vib. }} \\
& \quad=f^{1,1} G_{1}^{2}+f^{2} G_{1,1}: C_{44}^{\text {vib. }}=f^{2} G_{1,1}, \\
& C_{111}^{\text {vib. }}=f^{1,1,1} G_{1}^{3}+3 f^{2,1} G_{2} G_{1}+f^{3} G_{3}, \\
& C_{112}^{\text {vib. }}=f^{1,1,1} G_{1}^{3}+f^{2,1}\left(2 G_{1,1}+G_{2}\right) G_{1}+f^{3} G_{2,1}, \\
& C_{123}^{\text {vib. }}=f^{1,1,1} G_{1}^{3}+3 f^{2,1} G_{1} G_{1,1}+f^{3} G_{1,1,1}, \\
& C_{144}^{\text {vib. }}=f^{2,1} G_{1} G_{1,1}+f^{3} G_{1,1,1},
\end{aligned}
$$

$$
C_{456}^{\text {vib. }}=f^{3} G_{1,1}: C_{166}^{\text {vib. }}=f^{2,1} G_{1} G_{1,1}+f^{3} G_{2,1},
$$

where

$$
\begin{aligned}
& G_{3}=2 Q\left(r_{1}\right)\left(30+30 q_{0}+9 q_{0}^{2}-q_{0}^{3}-q_{0}^{4}\right) H+2 G_{2,1} \\
& G_{2,1}=Q\left(r_{2}\right)\left(15 \sqrt{2+1} 15 q_{0}+\frac{9}{q_{0}^{2} \sqrt{2}}-q_{0}^{3}-\sqrt{2} q_{0}^{4}\right) H, \\
& G_{2}=2 Q\left(r_{0}\right)\left(-6-6 q_{0}-q_{0}^{2}+q_{0}^{3}\right) H+2 G_{1,1}, \\
& G_{1,1}=Q\left(r_{2}\right)\left(-3 \sqrt{2}-6 \sqrt{2} q_{0}-\sqrt{2} q_{0}^{2}+2 q_{0}^{3}\right) H \\
& G_{1}=2 Q\left(r_{0}\right)\left(2+2 q_{0}-q_{0}^{2}\right) \\
& \quad+Q\left(r_{2}\right)\left(\sqrt{2}+2 q_{0}-\sqrt{2} q_{0}^{2}\right) H
\end{aligned}
$$

where

$$
Q\left(r_{1}\right)=A \exp \left(-\frac{r_{0}}{q}\right)
$$

and

$$
\begin{aligned}
& Q\left(r_{2}\right)=A \exp \left(-\frac{r_{0} \sqrt{2}}{q}\right), \\
& f^{2}=f^{3}=\frac{\hbar w_{0}}{8 r_{0}^{3}} \operatorname{coth} X, \\
& f^{1,1}=f^{2,1}=-\frac{1}{2 r_{0}^{3}} \frac{\hbar w_{0}}{48} \frac{X}{\sinh ^{2} X}+\operatorname{coth} X, \\
& f^{1,1,1}=\left(\frac{\hbar w_{0}}{384 r_{0}^{3}}\right)\left[\left(\frac{\hbar w_{0}}{k T}\right)^{2} \frac{\operatorname{coth} X}{6 \sinh ^{2} X}\right. \\
& \left.\sinh ^{2} X+\operatorname{coth} X\right], \\
& X=\frac{\hbar w_{0}}{2 k T}, \hbar=\frac{h}{2 \pi}, \quad k \text { is Boltzmann constant, } \\
& H=\left[\left(\frac{r_{0}}{q-2}\right) Q\left(r_{1}\right)+2\left(\frac{r_{0}}{q}-\sqrt{2}\right) Q\left(r_{2}\right)\right]^{-1} .
\end{aligned}
$$

\subsection{Acoustic attenuation}

Acoustic attenuation due to interaction of acoustical phonons and lattice phonons (i.e. phonon-phonon interaction) for longitudinal and shear wave are given by [23-26]:

$$
\begin{aligned}
{\left[(\alpha)_{\mathrm{p}-\mathrm{p}}\right]_{l} } & =\frac{E_{0} \omega^{2}\left(\Gamma_{l} / 3\right) \tau_{l}}{2 \rho V_{l}^{3}}, \\
{\left[(\alpha)_{\mathrm{p}-\mathrm{p}}\right]_{s} } & =\frac{E_{0} \omega^{2}\left(\Gamma_{s} / 3\right) \tau_{s}}{2 \rho V_{s}^{3}},
\end{aligned}
$$

where $\omega$ is angular frequency, $E_{0}$ is energy density, $\Gamma$ is acoustic coupling constant, $\tau$ is thermal relaxation time, $\rho$ is density and $V_{l}$ and $V_{s}$ are the velocities for longitudinal and shear wave, respectively.

Attenuation due to thermoelastic loss is given by [23-26]: 


$$
\left[(\alpha)_{\mathrm{th}}\right]_{l}=\frac{\omega^{2}\left\langle\gamma_{i}^{j}\right\rangle^{2} K T}{2 \rho V_{l}^{5}} .
$$

This type of attenuation occurs due to longitudinal wave only, because, due to shear wave propagation, the volume remains unaltered and there is no heating effect.

Acoustic coupling constant is given by [23-26]:

$$
\Gamma=9\left\langle\left(\gamma_{i}^{j}\right)^{2}\right\rangle-\frac{3\left\langle\gamma_{i}^{j}\right\rangle^{2} C_{v} T}{E_{0}} .
$$

$\left\langle\left(\gamma_{i}^{j}\right)^{2}\right\rangle$ and $\left\langle\gamma_{i}^{j}\right\rangle^{2}$ are square average and average square Grüneisen parameters corresponding to a particular direction of propagation and polarization.

Thermal relaxation time is given by [23-26]:

$$
\tau=\tau_{s}=\frac{\tau_{l}}{2}=\frac{3 K}{C_{v}\langle V\rangle^{2}},
$$

where $K$ is thermal conductivity, $C_{v}$ is specific heat per unit volume and $\langle V\rangle$ is the Debye average velocity.

The Debye average velocity is given by

$$
\frac{3}{(\langle V\rangle)^{3}}=\frac{1}{V_{l}^{3}}+\frac{2}{V_{s}^{3}} \text {. }
$$

Viscous drag coefficients due to screw and edge dislocations are given by [23-26]:

$$
\begin{aligned}
& \delta_{\text {screw }}=0.071 \chi, \\
& \delta_{\text {edge }}=0.053 \chi\left(1-\sigma^{2}\right)+0.0079 /\left(1-\sigma^{2}\right)(\zeta / \lambda) \eta
\end{aligned}
$$

where $\eta=\chi_{l}-(4 / 3) \chi_{s}, \chi_{l}=E_{0} \Gamma_{l} \tau / 3, \chi_{s}=E_{0} \Gamma_{s} \tau / 3$, $\lambda=\left(C_{11}+2 C_{12}\right) / 3, \zeta=\left(C_{11}-C_{12}+C_{44}\right) / 3$, and $\sigma=C_{12} /\left(C_{11}+C_{12}\right)$, and where $\lambda, \zeta, \eta, \sigma$ and $\chi$ are the bulk modulus, shear modulus, phonon viscosity, Poisson's ratio and compressional viscosity, respectively.

In presence of external magnetic field, magnon-phonon interaction also produces the acoustical dissipation in ferromagnetic and antiferromagnetic materials. Acoustic wave attenuation due to magnon-phonon interaction for longitudinal and transverse waves are given by [15]:

$$
\begin{gathered}
\alpha_{(m-p) l}=\frac{1}{2^{5}} \frac{\hbar \omega}{\pi \rho V_{l}} \frac{1}{\Theta_{\mathrm{C}}^{3} a^{4}}\left[\beta_{2}^{2}\left(\frac{\Theta_{0}^{2}}{4 \Theta_{\mathrm{C}}}+\hbar \gamma H_{0}\right)\right. \\
+2 \beta_{1} \beta_{2} \frac{\Theta_{0}^{2}}{4 \Theta_{\mathrm{C}}}\left(\frac{\Theta_{0}^{2}}{4 \Theta_{\mathrm{C}}}+\hbar \gamma H_{0}\right) \\
\left.+\beta_{1}^{2} \frac{\Theta_{0}^{4}}{2^{4} \Theta_{\mathrm{C}}^{2}}\right] \mathrm{e}^{-\left(\frac{\Theta_{0}^{2}}{4 \Theta_{\mathrm{C}} T}+\frac{\hbar \gamma H_{0}}{T}\right)}, \\
\alpha_{(m-p) t}=\frac{1}{2^{7}} \frac{\beta_{1}^{2}}{\pi \rho V_{t} a^{3}} \frac{\Theta_{0}^{3}}{\Theta_{\mathrm{C}}^{3}} \omega T \mathrm{e}^{-\left(\frac{\Theta_{0}^{2}}{4 \theta_{\mathrm{C}} T}+\frac{\hbar \gamma H_{0}}{T}\right)},
\end{gathered}
$$

where $H_{0}$ is the magnitude of applied field, $\gamma=$ gyromagnetic ratio, $\rho$ is the density, $\Theta_{0}$ and $\Theta_{\mathrm{C}}$ are temperatures evaluated using expressions given in Ref. [15], $\beta_{1}$ and $\beta_{2}$ are some constants. $V_{l}$ and $V_{t}$ are ultrasonic velocities of longitudinal and transverse wave, $a$ is lattice parameter, $\omega=2 \pi f=$ angular frequency of acoustical wave.

\section{Results and discussions}

SOEM and TOEM and bulk modulus of uranium monopnictides were evaluated using electrostatic and the Born repulsive potentials. Nearest neighbor distance and hardness parameter were used as input data. SOEM and bulk modulus and TOEM thus obtained, have been given in Tables I and II, respectively. SOEM and bulk modulus have been compared with available values in literature $[7,11,27,28]$. Our values are in good agreement with the available values. However, TOEM could not be compared due to lack of available values in literature. TOEM have been evaluated for LiF using present approach and values so obtained have been compared with available values in literature for LiF [22]. From Table II, it can be seen that the TOEM for LiF obtained using present approach are in good agreement with available values. Therefore, present approach of calculation for SOEM and TOEM for uranium monopnictides is justified.

TABLE I

Calculated, experimental and other papers second-order elastic moduli and bulk modulus $\left(10^{11} \mathrm{dyne} / \mathrm{cm}^{2}\right)$ of uranium monopnictides.

\begin{tabular}{l|c|c|c|c}
\hline \hline Comp. & $C_{11}$ & $C_{12}$ & $C_{44}$ & $\lambda$ \\
\hline UN & & & & \\
present & 42.00 & 4.90 & 5.23 & 17.3 \\
other & $42.0 \pm 0.4^{a}$ & $9.0 \pm 0.5^{a}$ & $7.9 \pm 0.08^{a}$ & $20 \pm 0.3^{a}$ \\
papers & - & - & - & $20^{d}, 19^{d}, 21^{d}$ \\
\hline UAs & & & & \\
present & 25.04 & 2.39 & 2.64 & 9.94 \\
other & $25.0 \pm 1^{a}$ & $1.0 \pm 1.5^{a}$ & $2.6 \pm 0.5^{a}$ & $9.0 \pm 1.3^{a}$ \\
papers & $25.0 \pm 1^{b}$ & $1.0 \pm 0.15^{b}$ & $2.6 \pm 0.5^{b}$ & $10^{d}, 9^{d}$ \\
& $25.0 \pm 1^{c}$ & $1.0 \pm 0.5^{c}$ & $2.6 \pm 0.5^{c}$ & $10.6^{d}$ \\
\hline USb & & & & \\
present & 16.03 & 1.88 & 2.04 & 6.59 \\
other & $16.0 \pm 1^{a}$ & $0.7 \pm 1.5^{a}$ & $2.0 \pm 1^{a}$ & $5.8 \pm 1.3^{a}$ \\
papers & $16.0 \pm 1^{b}$ & $2.0 \pm 1^{b}$ & $0.7 \pm 0.15^{b}$ & $6.2^{d}, 7.3^{d}$ \\
& $16.0 \pm 1^{c}$ & $0.7 \pm 0.15^{c}$ & $2.0 \pm 1.0^{a}$ & $5.5^{d}, 8.4^{d}$ \\
\hline \multicolumn{7}{l}{ Ref. [7]; ${ }^{b} \operatorname{Ref}[11] ;{ }^{c} \operatorname{Ref}[26] ;{ }^{d} \operatorname{Ref}[27]$} \\
\end{tabular}

${ }^{a}$ Ref. [7]; ${ }^{b}$ Ref. [11]; ${ }^{c}$ Ref. [26]; ${ }^{d}$ Ref. [27]

Lattice energy density $\left(E_{0}\right)$ and specific heat $\left(C_{v}\right)$ were obtained as function of the Debye temperature, $\Theta_{\mathrm{D}}[29,30]$ and are given in Table III. Square average Grüneisen parameters, average square Grüneisen parameters and acoustic coupling constants along [100] and [110] directions of propagation (along [001] and [110] directions of polarization for shear modes) are presented in Table IV. These values are similar to other fcc crystals [31-33]. Viscous drag coefficient due to screw and edge dislocations was evaluated using Eqs. (10) and (11) and is given in Table $\mathrm{V}$.

Attenuation coefficient due to magnon-phonon interaction for longitudinal and transverse waves has been evaluated by taking the value of $\gamma=1.76 \times 10^{7} \mathrm{Oe}^{-1} \mathrm{~s}^{-1}$ 
TABLE II

Third-order elastic constants $\left(10^{12}\right.$ dyne $\left./ \mathrm{cm}^{2}\right)$ of the materials at $300 \mathrm{~K}$.

\begin{tabular}{l|c|c|c|c|c}
\hline \hline Comp. & $C_{111}$ & $C_{112}$ & ${ }^{*} C_{123}=C_{144}$ & $C_{166}$ & $C_{456}$ \\
\hline UN & -88.61 & -1.45 & 0.98 & -1.95 & 0.91 \\
UAs & -56.69 & -0.56 & 0.50 & -0.96 & 0.50 \\
USb & -33.53 & -0.53 & 0.43 & -0.76 & 0.31 \\
LiF & -6.75 & -2.60 & 1.01 & -2.80 & 0.98 \\
Ref. [21] & -5.97 & -2.61 & 0.91 & -2.64 & 0.88 \\
\hline
\end{tabular}

${ }^{*} C_{123}=C_{144}$, since vibrational part is the same

\section{TABLE III}

Debye temperature $\Theta_{\mathrm{D}}$, phonon energy density $\mathrm{E}_{0}\left(10^{8} \mathrm{erg} /(\mathrm{mol} \mathrm{K})\right)$, specific heat $C_{v}\left(10^{7} \mathrm{erg} /(\mathrm{mol} \mathrm{K})\right)$, and Debye average velocity $\langle V\rangle\left(10^{5} \mathrm{~cm} / \mathrm{s}\right)$ for uranium monopnictides.

\begin{tabular}{l|c|c|c|c}
\hline \hline Comp. & $\Theta_{\mathrm{D}}[\mathrm{K}]$ & $E_{0}$ & $C_{v}$ & $\langle V\rangle$ \\
\hline $\mathrm{UN}$ & 249 & 5.56 & 2.41 & 2.1742 \\
$\mathrm{UAs}$ & 221 & 5.80 & 2.42 & 1.7844 \\
$\mathrm{USb}$ & 168 & 6.02 & 2.45 & 1.6185
\end{tabular}

TABLE IV

Square average and average square Grüneisen number for longitudinal $\left\langle\gamma_{i}^{j 2}\right\rangle_{l},\left\langle\gamma_{i}^{j}\right\rangle_{l}^{2}$ and shear $\left\langle\gamma_{i}^{j}\right\rangle_{s}^{2},\left\langle\gamma_{i}^{j}\right\rangle_{s^{*}}^{2}$ waves, acoustic coupling constants $\Gamma_{l}, \Gamma_{s}$, and $\Gamma_{s^{*}}$ at $300 \mathrm{~K}$ for uranium monopnictides.

\begin{tabular}{c|c|c|c|c|c|c|c|c}
\hline \hline & $\operatorname{Dir}^{n}$ & $\left\langle\gamma_{i}^{j 2}\right\rangle_{l}$ & $\left\langle\gamma_{i}^{j}\right\rangle_{l}^{2}$ & $\left\langle\gamma_{i}^{j}\right\rangle_{s}^{2}$ & $\left\langle\gamma_{i}^{j}\right\rangle_{s^{*}}^{2}$ & $\Gamma_{l}$ & $\Gamma_{s}$ & $\Gamma_{s^{*}}$ \\
\hline \multirow{4}{*}{ UN } & {$[100]$} & 8.22 & 1.66 & 0.08 & - & 67.62 & 0.78 & - \\
& {$[110]$} & 7.72 & 2.12 & 0.25 & 16.94 & 58.66 & 2.27 & 152.52 \\
\multirow{4}{*}{ USs } & {$[100]$} & 9.95 & 1.77 & 0.08 & - & 82.91 & 0.74 & - \\
& {$[110]$} & 8.96 & 2.25 & 0.18 & 20.74 & 72.15 & 1.70 & 186.72 \\
& {$[100]$} & 8.02 & 1.64 & 0.08 & - & 66.24 & 0.78 & - \\
& {$[110]$} & 7.22 & 2.04 & 0.26 & 16.59 & 57.41 & 2.40 & 149.35 \\
\hline
\end{tabular}

${ }^{l}$ for longitudinal wave; ${ }^{s}$ for shear wave, polarized along

$[001] ;{ }^{*}$ for shear wave, polarized along $[1 \overline{1} 0]$

TABLE V

Viscous drag coefficient due to screw and edge dislocation for uranium monopnictides at $300 \mathrm{~K}$ for longitudinal (in $\mathrm{cp}$ ) and shear (in $\mathrm{mp}$ ) waves.

\begin{tabular}{l|c|c|c|c|c}
\hline \hline Comp. & $\operatorname{Dir}^{n}$ & $\left(\delta_{\text {screw }}\right)_{\text {long }}$ & $\left(\delta_{\text {screw }}\right)_{\text {shear }}$ & $\left(\delta_{\text {edge }}\right)_{\text {long }}$ & $\left(\delta_{\text {edge }}\right)_{\text {shear }}$ \\
\hline UN & {$[100]$} & 3.37 & 0.38 & 2.85 & 3.52 \\
& {$[110]$} & 2.92 & 1.13 & 2.46 & 3.41 \\
UAs & {$[100]$} & 3.34 & 0.29 & 2.82 & 3.36 \\
& {$[110]$} & 2.91 & 0.68 & 2.45 & 3.19 \\
USb & {$[100]$} & 2.26 & 0.26 & 1.19 & 2.25 \\
& {$[110]$} & 1.96 & 0.82 & 1.65 & 2.32
\end{tabular}

at $f=1 \mathrm{GHz}$ and $T=10 \mathrm{~K}$ using Eqs. (12) and (13) for these materials and values are shown in Table VI along with attenuation values for yttrium iron garnet (YAG). $V_{l}$ and $V_{t}$ have been evaluated using elastic constants, density and lattice parameters $(a=4.89,5.779$, and $6.19 \AA$ for UN, UAs, and USb, respectively). These values for uranium monopnictides are smaller than that of YAG. ¿From Table VI and values of $\left(\alpha / f^{2}\right)$ shown in Figs. 1 and 2 , it can be seen that contribution to the attenuation by magnon-phonon interaction for longitudinal and transverse waves is negligible compared to contribution due to phonon-phonon interaction.

\section{TABLE VI}

Attenuation due to magnon-phonon interaction $(\mathrm{dB} / \mu \mathrm{s})$ for longitudinal and shear wave for UN, UAs and USb along with YAG.

\begin{tabular}{l|c|c}
\hline \hline Comp. & Longitudinal & Shear \\
\hline UN & $1.03 \times 10^{-8}$ & $3.47 \times 10^{-9}$ \\
UAs & $1.08 \times 10^{-8}$ & $4.32 \times 10^{-10}$ \\
USb & $7.76 \times 10^{-9}$ & $3.39 \times 10^{-10}$ \\
YAG & $1 \times 10^{-8}$ & $6 \times 10^{-6}$
\end{tabular}

Figures 1 and 2 show the temperature variation of acoustic attenuation, $\left(\alpha / f^{2}\right)$ for longitudinal and shear waves, respectively. From these figures it can be seen that

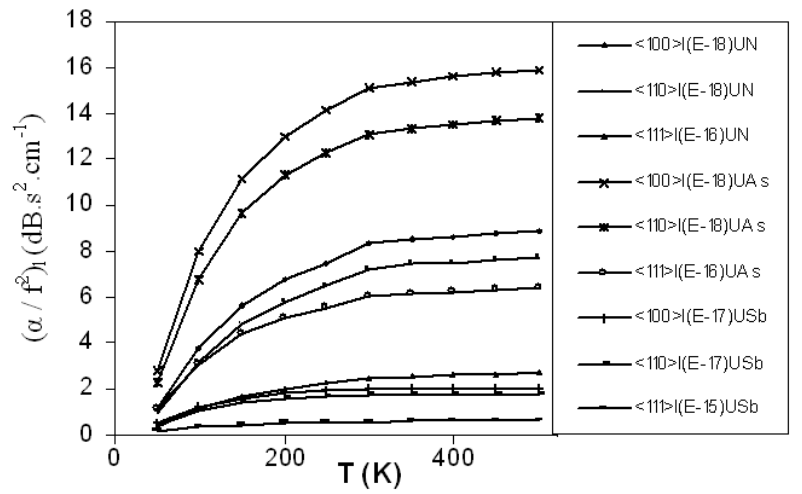

Fig. 1. Temperature variation of $\left(\alpha / f^{2}\right)_{l}$ along different directions of propagation for uranium monopnictides.

at lower temperatures, acoustic attenuation increases with temperature at faster rate and at higher temperatures rate of variation becomes nearly constant. The factor $V^{2} \tau$ (called diffusion coefficient) measures the ability of thermal phonons to absorb energy from a sound wave of velocity $V$ (in crystals energy is carried by phonon wave packets with group velocity $V)[34,35]$. From Fig. 3 it can be seen that $V^{2} \tau$ has larger value at lower temperature and decays exponentially. Therefore, at lower temperature range, the rate of absorption of sound en- 


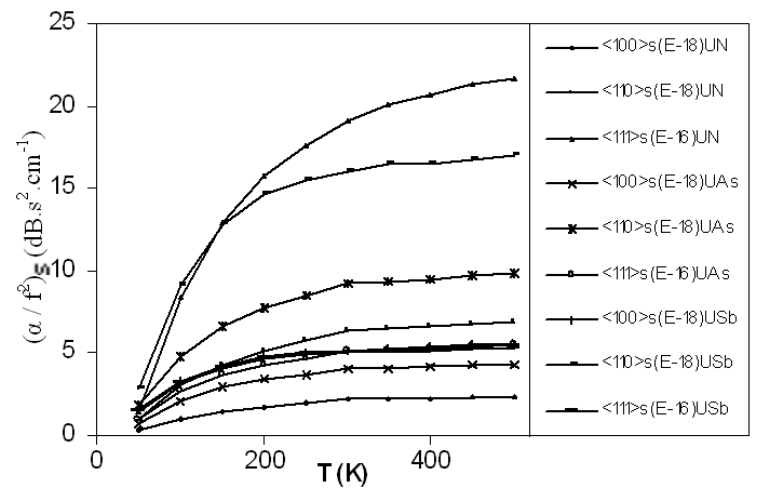

Fig. 2. Temperature variation of $\left(\alpha / f^{2}\right)_{s}$ along different directions of propagation for uranium monopnictides.

ergy (hence ultrasonic attenuation) is large and at higher temperatures it decreases and becomes nearly constant.

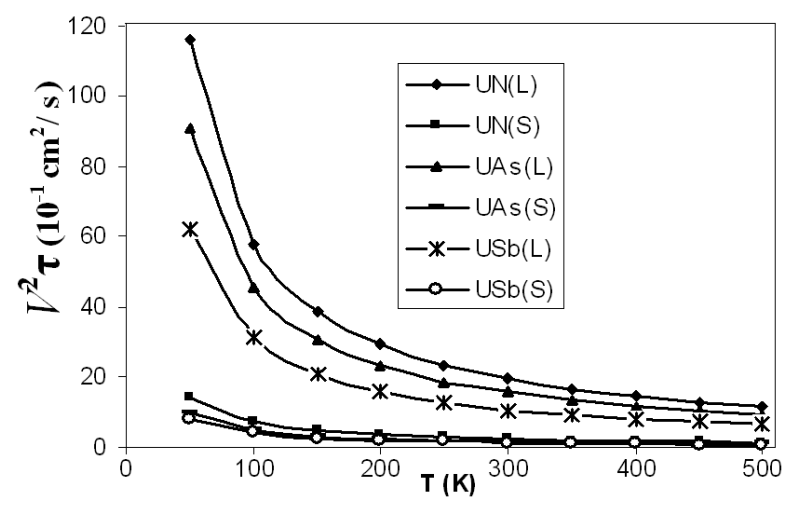

Fig. 3. Temperature variation of $V^{2} \tau$ for uranium monopnictides for longitudinal and shear waves.

The temperature variation of acoustic attenuation due to thermoelastic loss, $\left(\alpha / f^{2}\right)_{\text {th }}$ has been shown in Fig. 4. At lower temperatures, $\left(\alpha / f^{2}\right)_{\text {th }}$ increases with temperature and then becomes nearly constant.

Figures 5-8 show the temperature variation of acoustic coupling constant for longitudinal and shear modes, along [100] and [110] directions of propagation. From Figs. 5 and 6 , it can be seen that acoustic coupling constant (which is the measure of the anharmonicity of crystal) for longitudinal wave increases with temperature at slower rate in the temperature range 50-200 K, and beyond $200 \mathrm{~K}$, rate of temperature variation becomes nearly constant. Acoustic coupling constant for shear wave $\left(\Gamma_{s}\right)$ remains almost constant (Figs. 7 and 8). Thus total acoustic attenuation is mainly affected by longitudinal wave attenuation due to larger values of $\Gamma_{l}$.

Ultrasonic attenuation due to phonon-phonon interaction for longitudinal and shear waves, i.e. $\left(\alpha / f^{2}\right)_{l}$ and $\left(\alpha / f^{2}\right)_{s}$ increase from UN to USb due to increase in the molecular weight or increase in the anion atomic num-

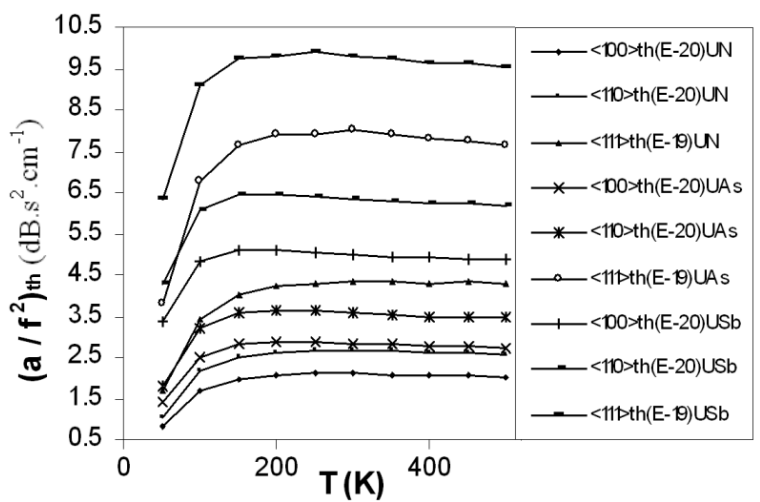

Fig. 4. Temperature variation of $\left(\alpha / f^{2}\right)_{\text {th }}$ along different directions of propagation for uranium monopnictides.

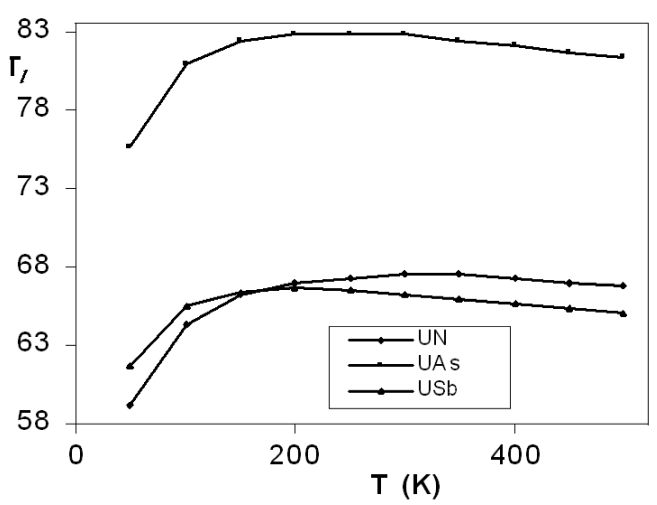

Fig. 5. Temperature variation of acoustic coupling constant $\left(\Gamma_{l}\right)$ for longitudinal wave along [100] direction of propagation for uranium monopnictides.

ber. As molecular weight increases, the velocity of anion reduces. Thus the Debye average velocity decreases from UN to USb (Table III).

From Table III, it can be seen that the Debye temperature decreases from UN to USb. Therefore, we can say that the larger the Debye temperature, the smaller will be attenuation. The Debye temperature depends on SOEM

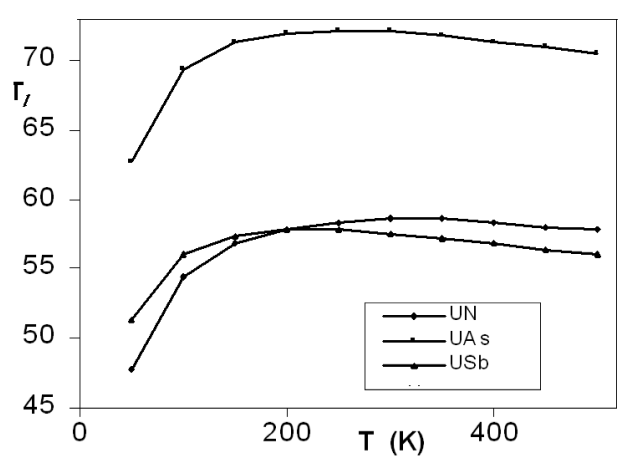

Fig. 6. As in Fig. 5, but along [110] direction. 


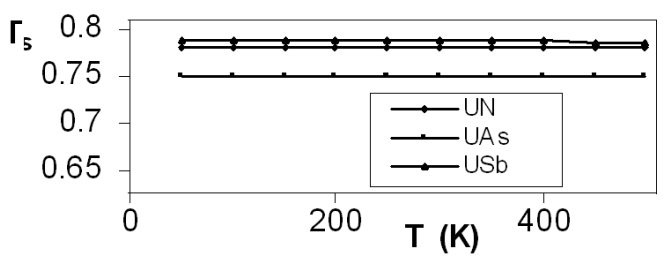

Fig. 7. Temperature variation of acoustic coupling constant $\left(\Gamma_{s}\right)$ for shear wave along [100] direction of propagation for uranium monopnictides.

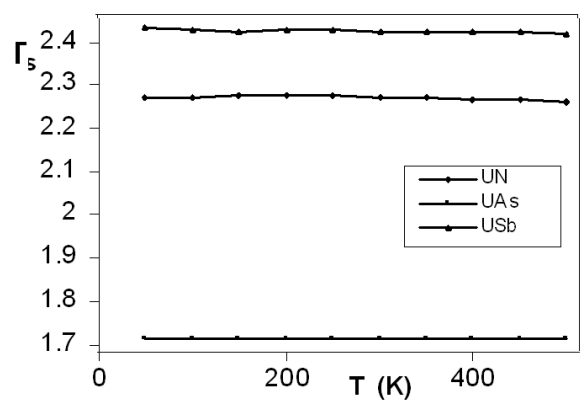

Fig. 8. As in Fig. 7, but along [110] direction.

values (through the Debye average velocity) hence, the larger the SOEM, the smaller the attenuation (because SOEM of the material are the measure of elasticity (or softening) of the crystal). Thus, it may be stated that USb is the most stable and has the least imperfections than the other two members of the group.

\section{Conclusion}

Total attenuation in these monopnictides in the present temperature range is the sum of attenuation due to phonon-phonon interaction, thermoelastic loss and dislocation damping due to screw and edge dislocations. Attenuation due to thermoelastic loss, dislocation damping and magnon-phonon interaction is negligible in comparison to the loss due to phonon-phonon interaction mechanism (Figs. 1 and 2, Table V and Table VI). Thus, phonon-phonon interaction is the principal cause dominating the acoustic attenuation due to larger value of $\Gamma_{l}$.

Using the polynomial fit law for $\left(\alpha / f^{2}\right)$, it has been found that attenuation due to phonon-phonon interaction and thermoelastic loss follow the fourth- and third-order polynomial temperature dependent fits, respectively in the temperature range $50-500 \mathrm{~K}$, i.e.

$$
\begin{aligned}
& \left(\alpha / f^{2}\right)_{\mathrm{p}-\mathrm{p}}=A+B_{1} T+B_{2} T^{2}+B_{3} T^{3}+B_{4} T^{4}, \\
& \left(\alpha / f^{2}\right)_{\mathrm{th}}=C+C_{1} T+C_{2} T^{2}+C_{3} T^{3},
\end{aligned}
$$

where $A, B_{1}, B_{2}, B_{3}, B_{4}$ and $\mathrm{C}, C_{1}, C_{2}, C_{3}$ are constants.

To the best of our knowledge, no experimental or theoretical data is available on attenuation for these uranium compounds, however, comparison has been made for SOEM and TOEM for LiF obtained using present approach and values available in literature. On the basis of good agreement for SOEM and TOEM data, it can be concluded that present approach for temperature dependence of acoustic attenuation is valid.

\section{Acknowledgments}

One of us (R.K.S.) is thankful to the University Grants Commission, New Delhi (Government of India) for financial assistance.

\section{References}

[1] J.S. Olsen, S. Stenstrup, L. Gerward, V. Bendict, J.C. Spirlet, G.D. Andrectt, J. Less-Common. Met. 98, 291 (1984).

[2] J. Neuenschwander, H. Boppart, J. Schoenes, E. Voit, O. Vogt, P. Wachter, in: Proc. Journées des Actinides Ed. J. Schoenes, Vol. 14, Zurich Edigenossiche Technische Hochschule, Zurich 1984, p. 30.

[3] C.F. Van Doorn, P. de V. Du Plessis, J. Magn. Magn. Mater. 5, 164 (1977).

[4] J.S. Olsen, L. Geward, V. Benedict, J. Appl. Crystallogr. 18, 37 (1985).

[5] M.S.S. Brooks, J. Phys. F 14, 633 (1984).

[6] M. Erbudak, F. Meier, Physica B 102, 134 (1980).

[7] J.A. Jackman, T.M. Holden, W.J.L. Buyers, P. de V. Du Plessis, O. Vogt, G. Gemossar, Phys. Rev. B 33, 7144 (1986).

[8] P.K. Jha, S.P. Sanyal, Phys. Rev. B 46, 3664 (1992).

[9] P.K. Jha, R.K. Singh, S.P. Sanyal, Physica B 174, 101 (1991).

[10] G.H. Lander, W.G. Stifling, J.M. Rossat-Mignod, M. Hagen, O. Vogt, Phys. Rev. B 41, 6899 (1990).

[11] P.K. Jha, S.P. Sanyal, Pramana J. Phys. 42, 9 (1994).

[12] T.M. Holden, W.J.L. Buyers, E.C. Svenssen, J.A. Jackman, A.F. Murray, O. Vogt, P. de V. Du Plessis, J. Appl. Phys. 53, 1967 (1982).

[13] J. Schoenes, B. Frick, O. Vogt, Phys. Rev. B 30, 6578 (1984).

[14] R.K. Singh, K.K. Pandey, Acta Phys. Pol. A 109, 219 (2006).

[15] A.E. Lord, Jr., Phys. Kondens. Materie 7, 232 (1968).

[16] G.G. Sahasrabudhe, S.D. Lambade, J. Phys. Chem. Solids 59, 789 (1998).

[17] S. Mori, Y. Hiki, J. Phys. Soc. Japan 45, 1449 (1978).

[18] K. Brugger, Phys. Rev. A 133, 1611 (1964).

[19] G. Leibfried, H. Hann, J. Phys. 150, 497 (1958).

[20] G. Leibfried, W. Ludwig, Solid State Physics, Vol. 12, Academic Press, New York 1961.

[21] Y. Hiki, Ann. Rev. Mater. Sci. Japan 11, 51 (1981).

[22] P.B. Ghate, Phys. Rev. B 139, A1666 (1965).

[23] S.K. Kor, U.S. Tondon, G. Rai, Phys. Rev. B 6, 775 (1972).

[24] R.K. Singh, R.P. Singh, M.P. Singh, P.C. Srivastava, J. Phys., Condens. Matter 20, 345227 (2008). 
[25] W.P. Mason, T.B. Bateman, J. Acoust. Soc. Am. 36 645 (1964).

[26] W.P. Mason, J. Acoust. Soc. Am. 42, 253 (1967).

[27] W.B. Stirling, G.H. Lander, O. Vogt, J. Phys. C, Solid State Phys. 16, 4093 (1983).

[28] L. Gerward, J.S. Olsen, U. Benedict, S. Dabos, J.P. Itié, O. Vogt, High Pres. Res. 1, 253 (1989).

[29] H. Rudigier, H.R. Ott, O. Vogt, Phys. Rev. B 32, 4584 (1985).

[30] Handbook of Chemistry and Physics, 83rd ed., Ed. D.R. Lide, R.C. Press, Florida 2002-2003.
[31] S.D. Lambade, Ph.D. Thesis, Nagpur University, Nagpur India 1997.

[32] G.G. Sahasrabudhe, S.D. Lambade, Acoust. Soc. Am. 104, (1998).

[33] S.D. Lambade, G.G. Sahasrabudhe, S. Rajagopalans, J. Phys. Chem. Solids 57, 217 (1996).

[34] V.L. Gurevich, Transport in Phonon System, North-Holland, Amsterdam 1986.

[35] J. Fabian, P.B. Allen, Phys. Rev. Lett. 82, 1478 (1999). 